\title{
Optimization of the Hollow Rectification Sill in the Forebay of the Pump Station Based on the PSO-GP Collaborative Algorithm
}

\author{
Wang Xi $(\mathbb{D}$, Weigang Lu $(\mathbb{D}$, Chuan Wang $(\mathbb{D}$, and Bo Xu $(\mathbb{i})$ \\ College of Hydraulic Science and Engineering, Yangzhou University, Yangzhou, Jiangsu 225009, China \\ Correspondence should be addressed to Weigang Lu; wglu@yzu.edu.cn
}

Received 18 November 2020; Revised 7 April 2021; Accepted 30 April 2021; Published 26 May 2021

Academic Editor: Zbigniew Krzemianowski

Copyright (c) 2021 Wang Xi et al. This is an open access article distributed under the Creative Commons Attribution License, which permits unrestricted use, distribution, and reproduction in any medium, provided the original work is properly cited.

\begin{abstract}
The layout of the pump station is easily affected by topography, site, and other factors, resulting in poor inlet flow patterns in the forebay, which seriously affect the normal operation of the pump station. To optimize its inlet flow pattern, the size of the hollow rectification sill has been continuously improved through physical model tests to meet the requirements of the required pump station inlet flow field. In this paper, particle swarm optimization (PSO) was combined with the Gaussian process (GP) to establish a particle swarm-Gaussian process (PSO-GP) model to predict the velocity uniformity of the inlet sump of pump stations with different hole-to-height ratios, hole-to-width ratios, upper-to-lower sill length ratios, and sill height-to-water table ratios. Finally, the hollow rectification sill with the optimal size was obtained and tested in the physical model to compare the rectification effect with other sizes of hollow sills. The results show that the algorithm model can help the traditional physical experiment quickly predict the velocity uniformity of the inlet sump of the pump station. Through the optimization by the PSO-GP algorithm, we can get the optimal size of the hollow rectification sill. Its hole-to-height ratio is 0.62 , its hole-to-width ratio is 0.37 , upper-to-lower sill length ratio is 0.63 , and sill height-to-water table ratio is 0.23 . It shows that this method is practical in the optimization design of the hollow rectification sill and provides a new method for the optimization of the flow field in the forebay of the pump station.
\end{abstract}

\section{Introduction}

To improve the unhealthy flow patterns such as vortex and deviation in the forebay and inlet sump of the pump station, various rectification measures [1], such as separate columns [2], separate sills [3], and diversion piers $[4,5]$, are usually taken in the forebay of the pump station to optimize the flow pattern of the forebay. Ying et al. [6] established a numerical model of the flow pattern in the forebay of the pump station. Based on computational fluid dynamics and the VOF method, they concluded that the optimization effect of the flow pattern was the best when the sills were installed at $80 \%$ of the distance between the column and the outlet. Xia et al. [7] used the N-S equation and reliable $\mathrm{k}-\varepsilon$ turbulence model to analyze the flow pattern of the forebay and inlet sump based on FLUENT. By constructing an inverted T-shaped sill and changing its parameters, they studied its influence on the flow pattern and side velocity of the forebay and found that the inverted T-shaped sill significantly improved the flow pattern of the forebay and obtained the optimal size through comparing at the same time. Luo et al. [8] used numerical simulation methods [9-15] to predict and simulate the flow in the forebay, used two different diversion piers to optimize the flow pattern in the forebay, and used model experiments to support each other. As a new rectification measure, the hollow sill [16] is more effective than other measures in rectifying the deviation, eliminating the vortex, clearing the sediment, and protecting the building.

In recent years, many scholars at home and abroad have applied intelligent algorithms to the optimization of pump stations [17, 18], such as genetic algorithm (GA) [19], ant colony optimization (ACO) [20], and wolf pack algorithm (WPA) [21]. Ahmadebrahimpour [22] applied the WPA to the optimal dispatch model of the reservoir and found that this algorithm can lead to simple results with a good optimization effect, which is suitable for optimal dispatch of the reservoir. Feng et al. [23] used the hybrid WPA to optimize the dispatch of the parallel pump station group, provided an 
effective operation plan, and reduced the operational energy consumption. Wang et al. [24] used genetic algorithms to establish a model with the minimum total operating power of the pump station as the objective function to optimize the plan to reduce the operating power consumption of the pump station. Shang et al. [25] used GA combined with the simulated annealing algorithm to optimize the load optimal dispatch of large hydropower plants. The particle swarm optimization (PSO) algorithm [26-31] has also been used in the pump station optimization in recent years [32] and has achieved good results, but this method requires the individual to be evaluated each time the individual position and velocity information are updated, which takes a long time [33], greatly increases the calculation cost, and affects the practicability of the optimization algorithm. Therefore, it is necessary to find a modeling solution to reduce individual evaluation time. The Gaussian process (GP) [34-36] is a rapidly developing machine learning method in recent years. It has good adaptability to complex problems such as small samples, high dimensionality, and nonlinearity. Studies have shown that the GP model greatly reduces the time required for accurate simulation in the optimal design of the flow pattern of the pump station while ensuring its accuracy.

In recent years, more and more scholars have combined multiple algorithms to optimize the model [37-41]. In this paper, we combined the advantages of the two algorithms and first established the PSO-GP model. Compared with the traditional GP model, this model used PSO to optimize the hyperparameters of the GP model [42] to avoid problems such as long training time in traditional GP modeling. Finally, the established PSO-GP model was used as the fitness evaluation plan of the PSO algorithm to optimize the hollow rectification sill of the forebay of the pump station. For the optimized rectification sill, physical model test $[43,44]$ and DPIV measurement technology were used [33, 45-47] to verify the validity and engineering practicability of the PSOGP model in optimizing the design of the hollow rectification sill of the forebay of the pump station.

\section{Principle of the PSO-GP Model}

2.1. Gaussian Process Model. The Gaussian process model can establish the mapping relationship between the input $x$ and the output $Y$ of the training set, and based on this mapping relationship, give the predicted value corresponding to the test sample $z+$. The Gaussian process describes a function distribution. It is a set where any subset of an infinite number of random variables conforms to the joint Gaussian distribution. Its properties can be determined by the mean function and the covariance function, namely,

$$
\left\{\frac{m(x)=E[f(x)]}{k\left(x, x^{\prime}\right)=E\{[f(x)-m(x)]\} \cdot\left[f\left(x^{\prime}\right)-m\left(x^{\prime}\right)\right]},\right.
$$

where $x, x^{\prime} \in R^{d}$ is an arbitrary $d$-dimensional vector and $m(x)$ and $k\left(x, x^{\prime}\right)$ represent the mean function and covariance function, respectively. Therefore, GP can be expressed as

$$
f(x) \sim P\left[m(x), k\left(x, x^{\prime}\right)\right] .
$$

Assuming a finite training set containing $n$ observations $D=\left(x_{i}, y_{i}\right)\{\mid i=1,2, \ldots, n\}=(X, y)$, among which $X=\left[x_{1}, x_{2}, \ldots, x_{n}\right]$ represents a $d \times n$-dimensional training input matrix composed of $n d$-dimensional training input vectors and $y=\left[y_{1}, y_{2}, \ldots, y_{n}\right]^{T}$ represents a corresponding training output vector composed of $n$ training output scalars $y_{i}$, the model can be expressed as

$$
y=f(x)+\varepsilon
$$

The observation target value $y$ is polluted by additive noise $\varepsilon$. $\varepsilon$ is a random variable that obeys the normal distribution, whose mean value is 0 and variance is $\sigma_{n}^{2}$, namely,

$$
\varepsilon \sim N\left(0, \sigma_{n}^{2}\right) .
$$

$X$ is the input vector, and $y$ is the observation value polluted by noise. The prior distribution of $y$ is

$$
y \sim N\left(0 . K+\sigma_{n}^{2} I\right)
$$

where $K=K(X, X)$ is the $n \times n$-order symmetric positivedefinite covariance matrix, matrix elements are used to measure the correlation between $x_{i}$ and $x_{j}$, and the joint Gaussian prior distribution composed of $n$ training sample outputs $y$ and $n^{*}$ test sample outputs $f^{*}$ is

$$
\left[\begin{array}{c}
y \\
f^{*}
\end{array}\right] \sim N\left(0,\left(\begin{array}{cc}
K(X, X)+\sigma_{n}^{2} I & K\left(X, X^{*}\right) \\
K\left(X^{*}, X\right) & K\left(X^{*}, X^{*}\right)
\end{array}\right)\right),
$$

where $K\left(X, X^{*}\right)$ is the $n \times n^{*}$-order covariance matrix between the $n^{*}$ test output sample and $n$ training samples and $K\left(X^{*}, X^{*}\right)$ is the $n^{*} \times n^{*}$-order covariance matrix of the test output sample itself.

The covariance function of GP must satisfy the Mercer condition: a nonnegative positive-definite covariance matrix can be guaranteed for any point set. Here, the square exponential covariance function (SE) is used:

$$
k_{\mathrm{SE}}\left(x, x^{\prime}\right)=\sigma_{f \mathrm{SE}}^{2} \exp \left[-\frac{r^{2}}{2}\right]
$$

where $\sigma^{2}$ is the amplitude factor and $r$ is the distance $\sqrt{\left(x-x^{\prime}\right)^{T} P\left(x-x^{\prime}\right)}$. The properties of the GP mean function and the covariance function are determined by a set of hyperparameters, which are also the only parameters that GP needs to determine. According to Bayes' theorem, based on the training set, the most likely output value corresponding to $x^{*}$ can be predicted. The purpose of adopting Bayes' theorem is to use the observed real data to continuously update the probability prediction distribution, that is, use the given input $x^{*}$, the input value $X$, and the observation target value $y$ of the training set, to infer the largest possible predicted posterior distribution of $y^{*}$ :

$$
y^{*} \mid x^{*}, X, y \sim N\left(m, \sum\right),
$$

where 


$$
m=K\left(X^{*}, X\right)\left(K(X, X)+\sigma_{n}^{2} I^{-1}\right) y,
$$

is the predicted mean value, showing the most likely test output value.

$$
\Sigma=K\left(X^{*}, X^{*}\right)-K\left(X^{*}, X\right)\left[K(X, X)+\sigma_{n}^{2} I\right]^{-1} K\left(X, X^{*}\right) .
$$

2.2. Particle Swarm Optimization Algorithm. The particle swarm optimization algorithm [48] has the advantages of easy implementation, simple algorithm, and fewer parameters and can effectively solve global optimization problems. The velocity and position update formula of the particle swarm algorithm is

$$
\begin{array}{r}
v_{i, d}^{k+1}=v_{i, d}^{k}+c_{1} \operatorname{Rand}\left(p_{i, d}^{k}-x_{i, d}^{k}\right)+c_{2} \operatorname{Rand}\left(p_{g, d}^{k}-x_{i, d}^{k}\right), \\
x_{i, d}^{k+1}=x_{i, d}^{k}+x_{i, d}^{k+1},
\end{array}
$$

where $c_{1}$ and $c_{2}$ are called the learning factor and acceleration constant; Rand () is a random number between $(0,1) ; x_{i, d}^{k+1}$ and $v_{i, d}^{k+1}$ are the velocity and position of the $d$-dimensional particle $i$ in $k$ iterations; $p_{i, d}^{k}$ is the position of the individual extreme value of the $d$-dimensional particle $i$; and $p_{g, d}^{k}$ is the position of the global extreme value of the group in the $d$ dimension.

2.3. PSO-GP Model. The traditional optimal hyperparameters of the GP model are obtained by the maximum likelihood estimation method, that is, the partial derivative of the hyperparameters is obtained by establishing the loglikelihood function of the conditional probability of the training sample, and then the conjugate gradient optimization method is used for the optimal hyperparameter solution. This optimization method will result in a large number of high-order calculations during the training of the GP model, which will affect the efficiency of GP modeling. In response to the above problems, this paper used the PSO intelligent algorithm to optimize the hyperparameters of the GP model to quickly establish the GP model. The number of hyperparameters of the GP model is determined by the selected covariance function and mean function. When initializing the particles, each particle represents a set of hyperparameters, which are sequentially assigned to the covariance function and mean function of the GP model. The mean absolute error (MAE) between the prediction of the GP model and the sample is used as the PSO fitness function for global optimization. The position and velocity information of the particles are updated in each iteration, and the calculation is stopped when the maximum number of iterations is reached.

For the traditional Gaussian process, the type of covariance function and the input dimension of the data determine the number of parameters of the GP model. Assume that there are $N$ groups of input data $1 \times n$, the corresponding output is $1 \times m$, and the Gaussian process covariance function is the exponential squared covariance function:

$$
k\left(x, x^{\prime}\right)=\sigma_{f}^{2} \exp \left[-0.5\left(x, x^{\prime}\right)^{T} M\left(x, x^{\prime}\right)\right] .
$$

Among them, matrix $M=\operatorname{diag}(l)$ is the positive featurelength scale parameter corresponding to the input variable element, and $\sigma_{f}^{2}$ is the signal covariance. For $N$ sets of training data, the training parameters required for the corresponding Gaussian process are

$$
\text { GP parameters }=1 \times n+1 .
$$

After initializing and establishing the GP model, assume that the predicted output value obtained by the GP model is $y_{\text {pred, } i}$, and the original accurate output value of the GP test input sample is $y_{\text {test, } i}$. Assuming that each set of data selects $N$ data points, the mean square error (MSE) formula is used here. The model design process is shown in Figure 1.

$$
E=\frac{1}{N} \sum_{i=1}^{N}\left(y_{\text {pred }, i}-y_{\text {test }, i}\right)^{2}
$$

\section{Hollow Rectification Sill of the Forebay in the Pump Station}

3.1. Model Building. The sill is currently widely used in engineering as a rectification measure with low cost and convenient construction. The commonly used sill is mostly an integral solid structure, and the flow velocity and direction of the water flow will be adjusted after passing through the rectification sill. However, when the water level is low, the commonly used rectification sill will cause the water level of the forebay to decrease, which will worsen the inlet flow pattern, and it is easy to form sedimentation behind the sill, which will affect the normal operation of the pump station. When the new hollow rectification sill is at a low water level, water can pass through the hollow part at the bottom, thereby minimizing the impact of the rectification sill on the overflowing water level, and at the same time, it is more beneficial to the adjustment of the flow velocity and flow direction. It also plays a certain role in scouring and silting before and after the rectification sill.

A geometric model of the water intake part of the pump station is established. The plane size is shown in Figure 2, and the unit of the size in the figure is $\mathrm{mm}$. The single pump flow of the pump station studied in this paper is $25 \mathrm{~m}^{3} /$ s, and the total flow is $100 \mathrm{~m}^{3} / \mathrm{s}$. The pump station is on the left bank of the diversion channel. The model is composed of the intake channel, the forebay of the pump station, and the inlet sump. To ensure similar flow patterns, we chose the upstream $800 \mathrm{~m}$ of the diversion channel to the intersection of the pump station and the check gate for our study. The pump station has four units, numbered 1-4 from right to left according to the flow direction. The elevation of the bottom of the intake channel is $-2 \mathrm{~m}$, the elevation of the base plate of the inlet flow channel is $-5.3 \mathrm{~m}$, and the elevation of the base plate of the gate is $-2 \mathrm{~m}$. The working condition with 
clc

clear

close all

(i) Normalized training input $x_{\text {train }}, \operatorname{train} x_{n}$

(ii) Normalized training output $y_{\text {train }}, \operatorname{train} y_{n}$

(iii) Initialize PSO

(iv) Setting parameters

$$
\left\{\begin{array}{l}
m \\
n \\
\text { number of iterations } \\
\text { acceleration constant } c_{1} \\
\text { acceleration constant } c_{2} \\
\text { the upper limit of search } \\
\text { the lower limit of search }
\end{array}\right.
$$

(v) Search GP hyperparameters for PSO in the global space:

(1) Initialize a set of initial values randomly to form a population

(2) Substitute the population into GP and predict the output GP.y

(3) Set PSO fitness function GP.error

$$
\text { GP.error }=\text { MSE }\left(\text { GP. } y-\operatorname{trainy}_{n}\right)
$$

(4) Comparison of fitness functions to obtain individual extreme values and multiple extreme values

(5) According to PSO optimization, change the position and speed of the flying race

(6) Update and record the current prediction optimal solution $x\left(i^{*}\right)$

(vi) Carry out PSO reorganization and save the maximum optimal solution $x\left(i^{*}\right)$

(vii) Reach the number of iterations and complete the PSO optimization algorithm

(viii) Use $x\left(i^{*}\right)$ as the GP hyperparameter and initialize GP

(ix) Normalize test input $x_{\text {test }}$ and substitute it into GP as input data

(x) Get the output GP.y, and use denormalization to get the optimal solution $x_{\text {best }}$ of $x_{\text {test }}$

End

(a)

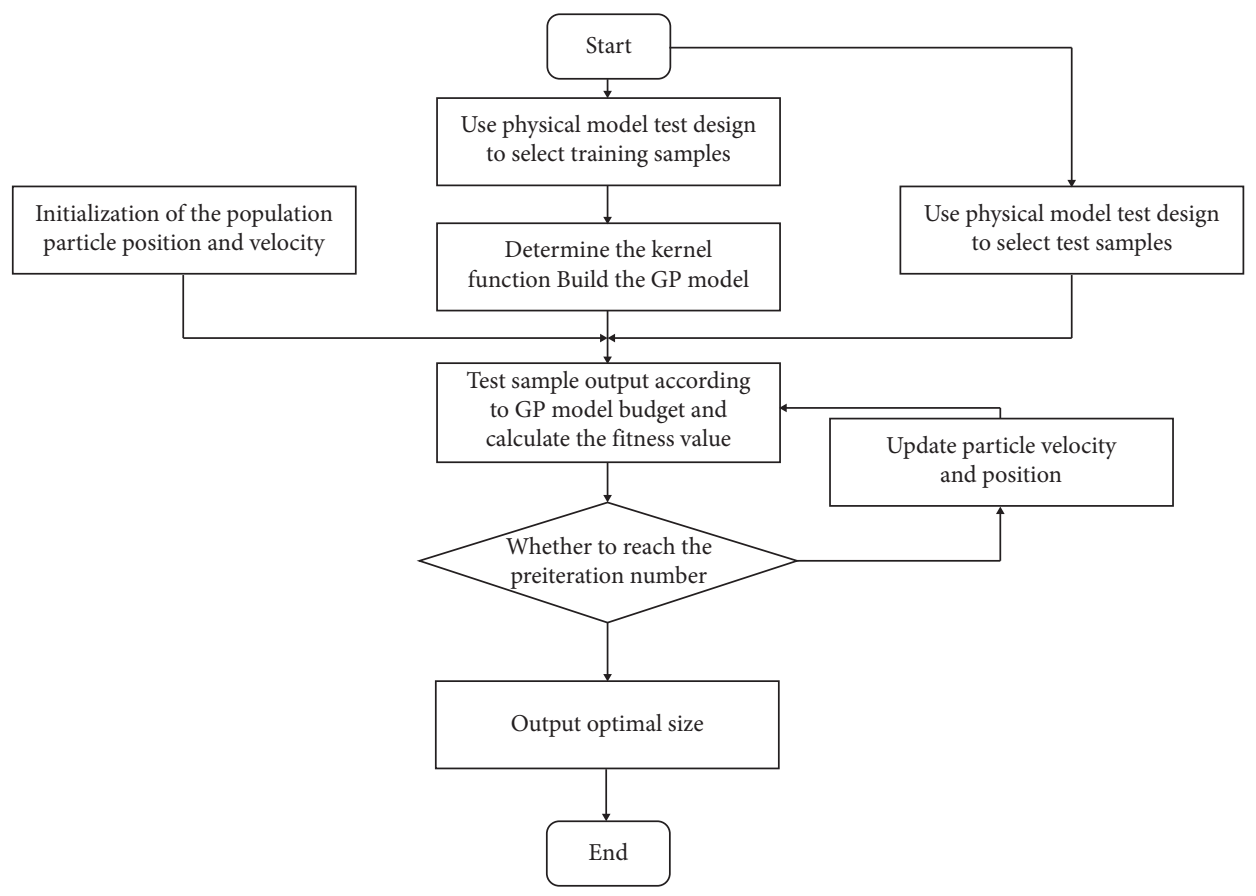

(b)

FIgURe 1: PSO-GP model. (a) PSO-GP pseudo-code. (b) PSO-GP flowchart.

$1.7 \mathrm{~m}$ of design water level and four units operating together is studied.

A physical model is built as shown in Figure 3. The total inflow is $100 \mathrm{~m}^{3} / \mathrm{s}$; except for the inlet and outlet and free water surface, the rest are solid walls. The scale of the model is $1: 25$. The entire test model includes the intake channel, the forebay, the pier, the inlet flow channel, the control valve, the water tank, the pipeline, and the auxiliary pump. At the same time, the WYG-III; wireless measurement and control intelligent water level meter of Nanjing Hydraulic Research Institute and piezometer tube were used to measure the water level and verify the accuracy of the water level. 


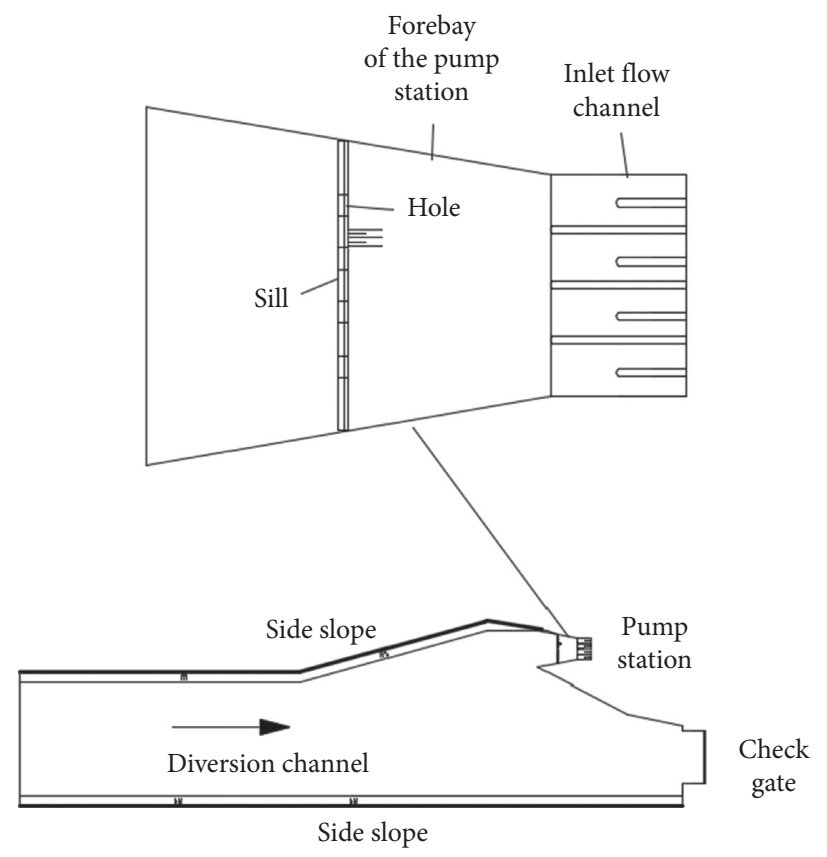

FIGURE 2: Schematic diagram of the water intake model of the pump station.

FLOWATCH portable hydrometric propeller was mainly used for the flow velocity measurement, and the LDY-250S integrated electromagnetic flow meter was adopted for flow measurement. The experimental flow field data were collected by the DPIV high-definition flow field measurement system. Digital particle image velocimetry (DPIV) is an emerging branch of PIV, which is a noninterference, transient, and full-field flow measurement technology developed by using image processing technology based on flow display. Then, they were processed by software Tecplot. The layout of the sill in the specific model is shown in Figure 4.

3.2. Type Selection of the Hollow Sill. The specific arrangement is to install a sill at the intersection of the forebay and intake channel with a height of $1 \mathrm{~m}$. Four holes shall be opened directly opposite to the four inlet flow channels on the rectification sill with the hole width of $2.5 \mathrm{~m}$ and the hole height of $0-1 \mathrm{~m}$. To make the test results universal, the ratio of the hole height to the sill height is defined as the hole-toheight ratio, the ratio of the total width of the four holes to the total width of the sill is the hole-to-width ratio, the ratio of the upper and lower bottom length of the sill is the upperto-lower sill length ratio, and the ratio of sill height to the water level is the sill height-to-water table ratio. The placement position of the sill is shown in Figure 4, and the detailed drawing of the hollow sill is shown in Figure 5.

To study the hollow rectification sill, 100 sets of randomly matched data with different hole-to-height ratios, hole-to-width ratios, upper-to-lower sill length ratios, and sill height-to-water table ratios were selected, numbered, and analyzed. See the specific size selection range in Table 1.

\section{Optimization Design of the Hollow Sill of the Forebay of the Pump Station Based on the PSO-GP Model}

4.1. Establish the Index of Flow Rate Uniformity. The hollow sill with the optimal size parameter (obtained through PSOGP optimization) was put into the physical model experiment for verification. The flow pattern was measured by DPIV, and finally, the velocity cloud diagram was obtained after Tecplot processing. The section at the junction of the forebay and the inlet flow channel is selected as the characteristic section (shown in Figure 4), and the uniformity of the axial velocity distribution $V_{a u}$ is used as the index for evaluating the quality of flow rate. The closer to $100 \% V_{a u}$, the better the velocity uniformity. The calculation formula is

$$
V_{a u}=\left[1-\sqrt{\sum_{i=1}^{n} \frac{\left(\left(V_{a i} / V_{a}\right)-1\right)^{2}}{n}}\right] \times 100 \% .
$$

The derived flow velocity data value is the average flow velocity of each small section after dividing the entire section into multiple small sections. For this purpose, an areaweighted uniformity objective function can be introduced:

$$
V_{a u}=\left[1-\sqrt{\frac{\sum_{i=1}^{n} A_{i}\left(V_{i a} / V_{a}\right)^{2}}{\sum_{i=1}^{n} A_{i}}}\right] \times 100,
$$

where $A_{i}$ is the area of each section, $\mathrm{m}^{2}, V_{a i}$ is the average axial velocity of each section, $\mathrm{m} / \mathrm{s}, n$ is the number of nodes, $V_{a u}$ is the uniformity of the axial velocity distribution, and $V_{a}$ is the average axial velocity of each section element, $\mathrm{m} / \mathrm{s}$, and its value is 


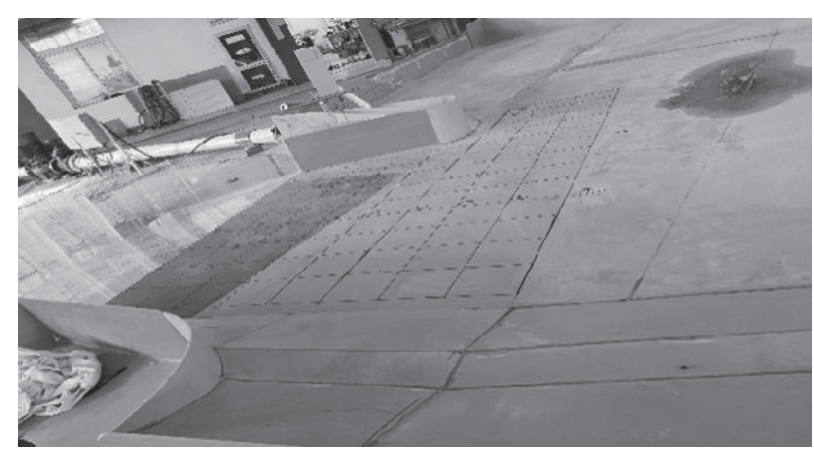

Figure 3: Physical model test device.

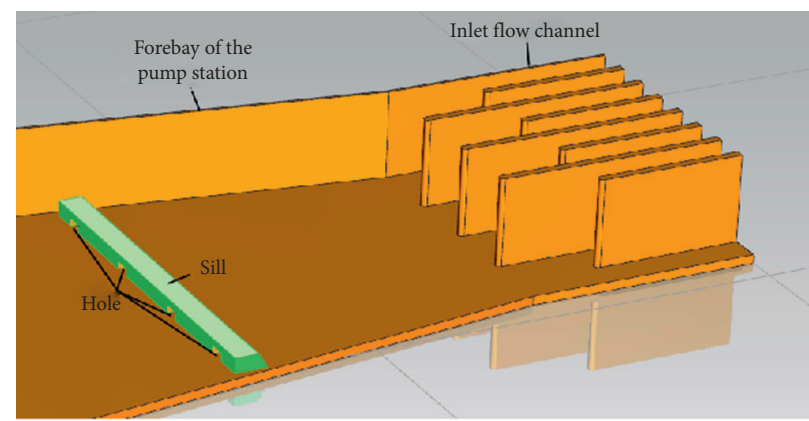

FIGURE 4: Schematic diagram of the sill position.

$$
V_{a}=\frac{\int_{s 1} V_{i}\left|\vec{V} \cdot \mathrm{d} \vec{A}_{i}\right|}{\int_{s 1}\left|\vec{V} \cdot \mathrm{d} \vec{A}_{i}\right|}=\frac{\sum_{i=1}^{n} u_{i} A_{i}}{\sum_{i=1}^{n} u_{i} A_{i}}
$$

4.2. PSO-GP Optimization Processing. After the PSO-GP model of the hollow sill of the forebay of the pump station is established, the PSO algorithm is used to optimize the design. The maximum number of iterations in the PSO algorithm is 1000, the acceleration constant is $c_{1}=1.7, c_{2}=1.5$, the inertia weight is $\omega=0.5$, the initial population is 20 , and the dimension is 4 . The optimized hollow sill is with the hole-to-height ratio of 0.62 , hole-towidth ratio of 0.37 , upper-to-lower sill length ratio of 0.63 , and sill height-to-water table ratio of 0.23 . It takes about 24 days to establish a physical model and conduct the test, about 500 seconds to complete the training of the PSO-GP model, 300 seconds to use PSO and PSO-GP to optimize the hollow sill of the forebay of the pump station, and 0.3 seconds for a generation of 20 particles (each particle represents 4 sets of size parameters). As a result, this far exceeds the time required to use other algorithms as the fitness function of the particle swarm algorithm. The process of bottom sill optimization is shown in Figure 6.

4.3. PSO-GP Optimization Error Analysis. We built a physical model for experimentation, selected 80 sets of data as training samples for the PSO-GP model, made hollow sills of different sizes based on these data, and conducted tests in the physical model. Then, we used DPIV to test and measure, observed the flow patterns affected by the hollow sills with different sizes, and used Tecplot software for postprocessing to obtain the corresponding velocity uniformity of the forebay of the pump station. And we used the velocity uniformity as the training input data of the PSO-GP model to establish the model; besides, we selected another 20 groups as the test samples, the output of the PSO-GP model was $y_{\text {pred, } i}$, and the output of each group of corresponding physical model test processing was $y_{\text {test }, i}$. To have a comprehensive understanding of the hollow sill, we set the holeto-height ratio to $0-1$, the hole-to-width ratio to $0.14-0.48$, the upper-to-lower sill length ratio to $0.4-1$, and the sill height-to-water level ratio to $0.1-0.5$. The error results are shown in Figure 7.

In order to verify the advantages of the PSO-GP algorithm, here we select the common PSO-GP algorithm and the Gaussian process algorithm for comparison and study, substitute the flow uniformity index as the training input data, respectively, and perform iterative calculations with different iteration times. The results are compared with the real values, and the results are shown in Figure 8. The calculation results show that compared with the PSO-GP algorithm and the Gaussian process algorithm, the PSO-GP algorithm has a significantly lower computational cost and smaller errors, especially the number of iterations. In the case of high accuracy can be achieved, using the described algorithm PSO-GP has a better advantage in the calculation time and accuracy. 


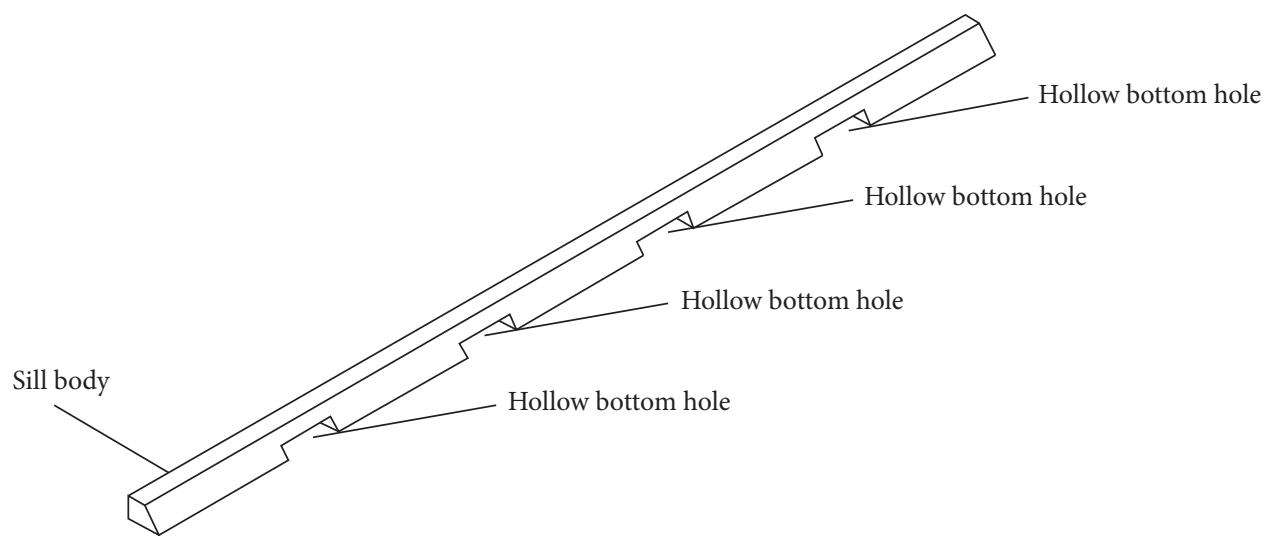

Figure 5: Detailed drawing of the hollow sill.

TABLE 1: Size selection range of hollow sills.

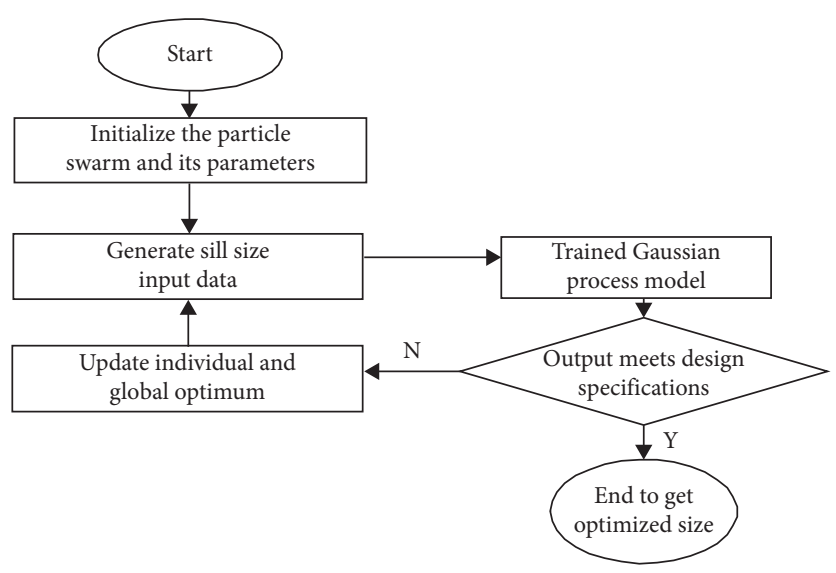

FIGURE 6: PSO-GP optimization flowchart.

\section{Comparative Analysis of Optimization Results}

After installing a hollow sill in the forebay of the pump station, water flowing out of the hollow part fully contacts water flowing through the rectification sill and energy dissipation behind the sill. The water flow in the hollow part has a good effect on the straight adjustment of the flow direction so that the water flow passing through the hollow part is relatively straight after adjustment, and the rectification sill plays a leading role in the adjustment of the entire flow. In the experiment, we found that the hole-to-height ratio, hole-to-width ratio, upper-to-lower sill length ratio, and sill height-to-water level ratio have a greater influence on the rectification effect. To study the influence of these factors on the flow pattern of the inlet water, plan 1 is the original plan without hollow sill rectification, and plans 2 to
7 are plans with six different sizes of the hollow sill. The flow fields of the surface layer and the bottom layer are shown in Figure 9. It can be seen from the figure that the hollow part gradually increases, the straight water flow it drives gradually increases, and the backflow of the forebay gradually decreases, but when the opening is too large, the hollow part of the sill will reduce the rectification effect of the water flow via holes; as a result, the rectification effect of the hollow rectification sill is reduced. From Figures $9(\mathrm{f})-9(\mathrm{~h})$, it can be seen that the backflow area on the right side of the diversion channel increases, and there is a local high-speed area on the right side of the no. 1 inlet flow channel. Through PSO-GP optimization, we can get that, for the best size, the hole-toheight ratio is 0.62 , the hole-to-width ratio is 0.37 , the upperto-lower sill length ratio is 0.63 , and the sill height-to-water level ratio is 0.23 . The surface layer and bottom layer flow field are shown in plan 8 , respectively. The flow pattern of the 


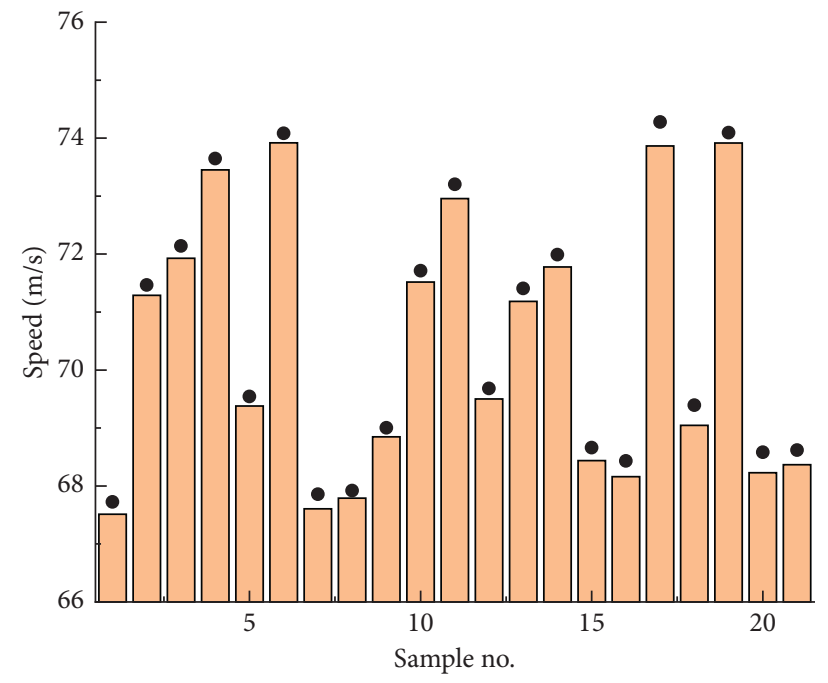

Actual value

- PSO-GP

Figure 7: PSO-GP optimization error table.

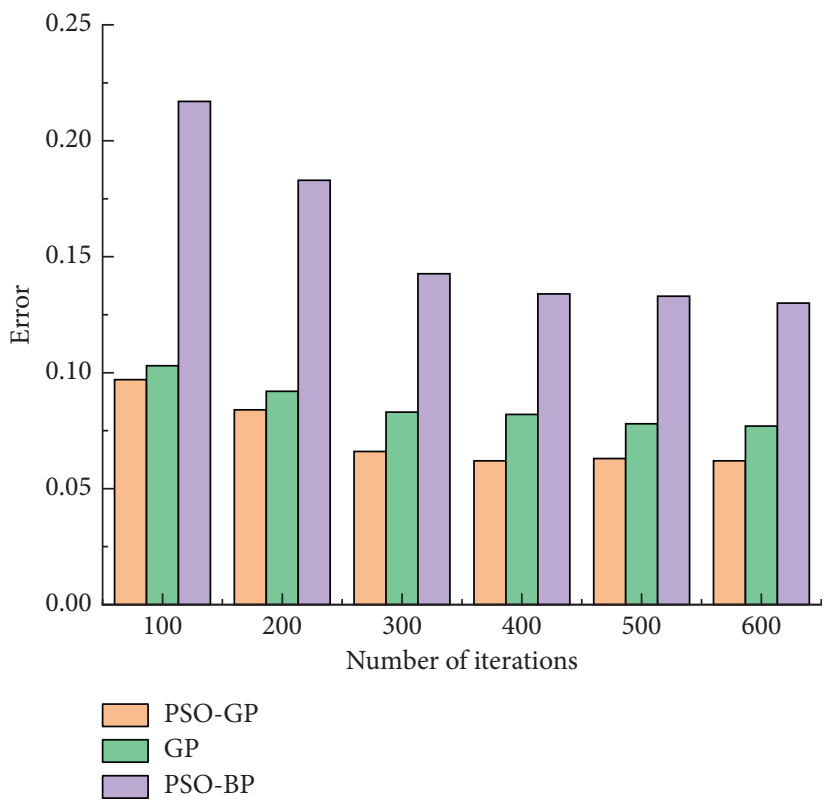

Figure 8: Comparison chart of different algorithms.

forebay is relatively good, and the flow velocity distribution in the inlet sump is also relatively uniform.

According to the flow velocity distribution of each inlet flow channel section, the flow velocity uniformity of the inlet section of each flow channel is studied and analyzed. According to formula (14), the velocity uniformity of each flow channel is calculated, and it can be seen that the velocity uniformity is $65 \%-75 \%$. When the hole-to-height ratio is less than or equal to 0.62 , the velocity uniformity increases with the increase of the hole-to-height ratio, so we can get that opening holes on the sill is beneficial to adjust the velocity uniformity. When the hole-to-height ratio is larger than 0.62 , the velocity uniformity gradually decreases and reaches the maximum when the hole-to-height ratio is 0.62 . Overall, the velocity is the most uniform when the hole-toheight ratio is 0.6 . When the hole-to-width ratio is less than or equal to 0.37 , the velocity uniformity increases with the increase of the hole-to-width ratio and gradually decreases when the hole-to-width ratio is larger than 0.37 . The velocity uniformity reaches the maximum when the hole-to-width ratio is 0.37 . Overall, the flow velocity is the most uniform when the hole-to-width ratio is 0.37 . When the upper-tolower sill length ratio is less than or equal to 0.63 , the velocity uniformity increases with the increase of the upper-to-lower sill length ratio. When the upper-to-lower sill length ratio is greater than 0.63 , the velocity uniformity gradually decreases 

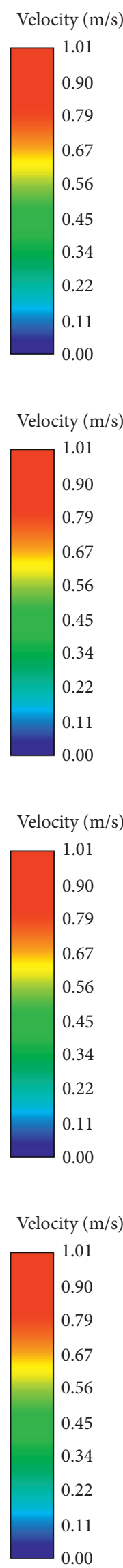

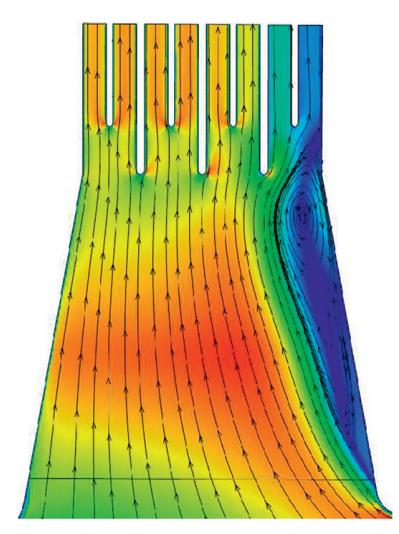

(a)

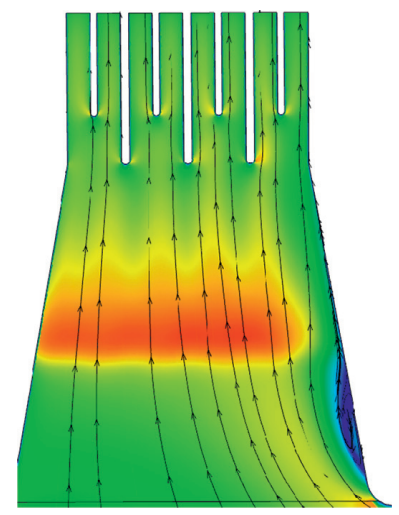

(c)

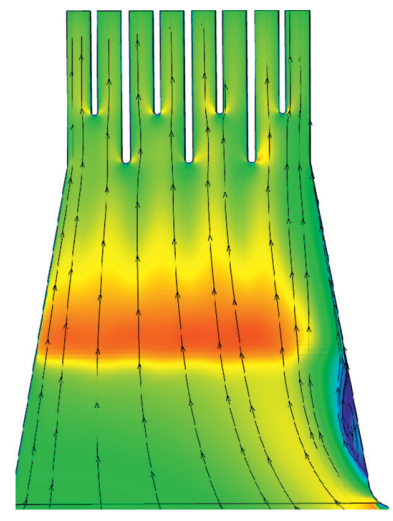

(e)

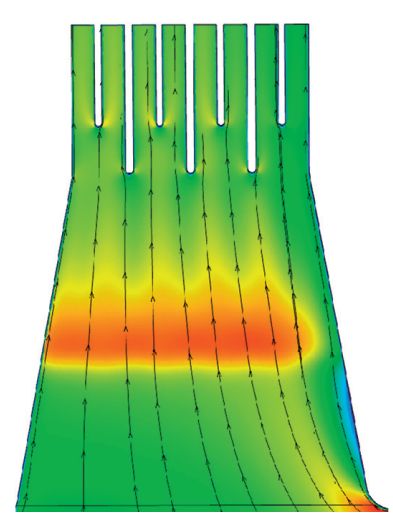

(g)
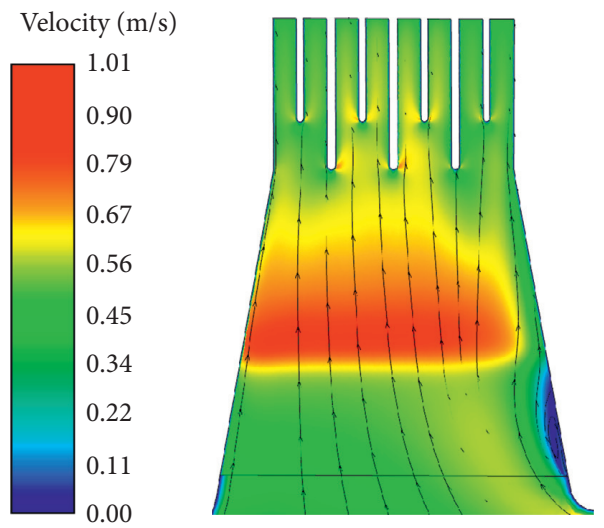

(b)
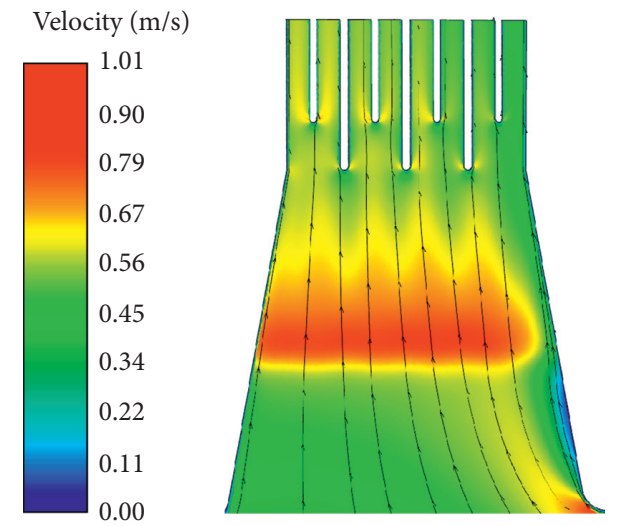

(d)
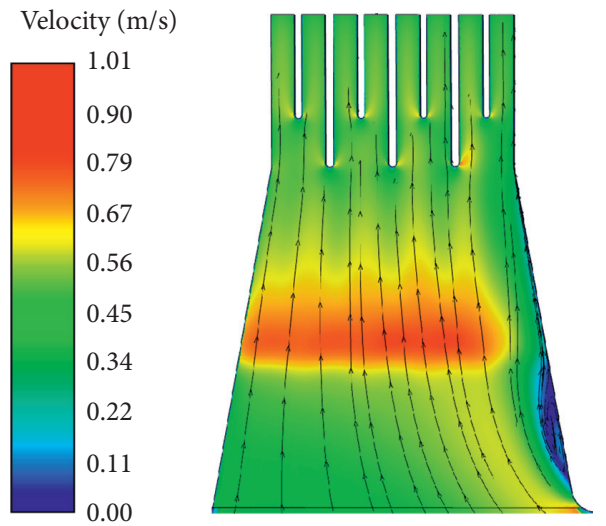

(f)
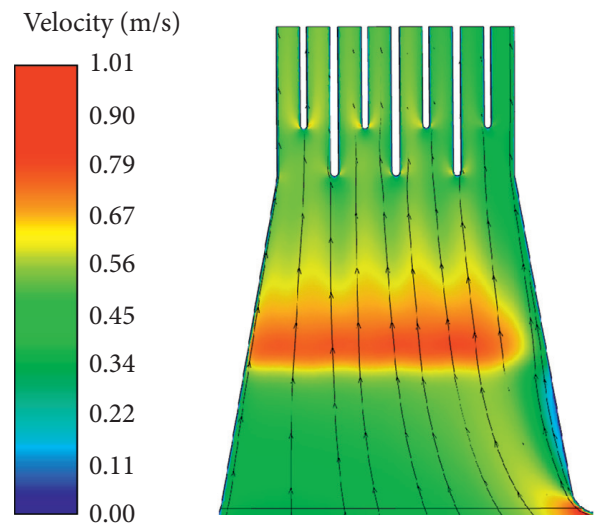

(h)

Figure 9: Surface flow field diagram of each plan: (a) plan 1, (b) plan 2, (c) plan 3, (d) plan 4, (e) plan 5, (f) plan 6, (g) plan 7, and (h) plan 8. 
and reaches the maximum when the ratio is 0.63 . Overall, the velocity is the most uniform when the upper-to-lower sill length ratio is 0.63 . The velocity uniformity increases with the increase of the hole-to-height ratio when the sill heightto-water table ratio is less than or equal to 0.23 , and it gradually decreases when the ratio is larger than 0.23 . The velocity uniformity reaches the maximum when the sill height-to-water level ratio is 0.23 . Overall, the velocity is the most uniform when the sill height-to-water level ratio is 0.23 .

\section{Conclusion}

In this paper, particle swarm optimization was combined with the Gaussian process to establish the PSO-GP model for the optimization of the design of the hollow sill of the forebay of the pump station, thereby effectively reducing the type selection time of the hollow sill during the optimization of the forebay of the pump station and also improving the accuracy of the type selection optimization. Through physical model test comparison, the PSO-GP model can be used to obtain more accurate velocity distribution of the forebay of the pump station, and velocity uniformity can be used to judge the optimization effect of the hollow sill. The optimal size of the hollow sill of the forebay of the pump station optimized by the PSO-GP model is as follows, that is, the hole-to-height ratio is 0.62 , the hole-to-width ratio is 0.37 , the upper-to-lower sill length ratio is 0.63 , and the sill height-to-water level ratio is 0.23 . After we put the hollow sill of this size in a physical model for tests and compared it with the rectification effect of other sizes of hollow sills, we found that the rectification effect of this size is the best, indicating that this method is practical in the optimization design of the hollow rectification sill of the forebay of the pump station.

\section{Data Availability}

The data used to support the findings of this study are available from the corresponding author upon request.

\section{Conflicts of Interest}

The authors declare that there are no conflicts of interest regarding the publication of this paper.

\section{Acknowledgments}

This research was funded by the National Natural Science Foundation of China (51779215 and 52079120) and Jiangsu Graduate Research and Innovation Program (KYCX20_2980).

\section{References}

[1] Z. Su, J. Lu, Z. Zhang, H. Li, and Z. Su, "Study on rectifier technology in forebay of pumping station," Yellow River, vol. 41, no. 1, pp. 69-75, 2020.

[2] X. Wu, Z. Peng, Q. Fu, Z. Zhong, and Y. Song, "Study on improvement measures of flow pattern in inlet water pumping station," Journal of Irrigation and Drainage Engineering, vol. 38, no. S2, pp. 96-100, 2019.

[3] J. Feng, Z. Li, and S. Qian, "Study on hydraulic characteristics of double-sill rectification downstream a sluice-pump station," International Journal Hydroelectric Energy, vol. 40, no. 1, pp. 25-31, 2020.

[4] J. Jia, J. Zhou, M. Zhao, J. Qin, C. Fu, and J. Lu, "Impact of wedge-shaped guide piers on rectification effect of large diffusion angle fore bay for pumping station," International Journal Hydroelectric Energy, vol. 37, no. 8, pp. 88-91, 2019.

[5] C. Zhang, C. Zhou, Y. Zhou, L. Xu, and L. Cheng, "Effect of geometric parameters of Y-shaped diversion piers on flow pattern in forebay of pumping station," South-to-North Water Transfers and Water Science \& Technology, vol. 18, no. 3, pp. 192-200, 2020.

[6] J. Ying, X. Yu, W. He, and J. Zhang, "Volume of fluid modelbased flow pattern in forebay of pump station and combined rectification scheme," Journal of Irrigation and Drainage Engineering, vol. 38, no. 5, pp. 476-480, 2020.

[7] C. Xia, L. Cheng, W. Jiao, and D. Zhang, "Numerical simulation on rectification measure of inverted T-shaped sill at forebay of pump station," South-to-North Water Transfers and Water Science \& Technology, vol. 16, no. 2, pp. 146-150+163, 2018.

[8] C. Luo, J. Qian, C. Liu, F. Chen, J. Xu, and Q. Zhou, "Numerical simulation and test verification on diversion pier rectifying flow in forebay of pumping station for asymmetric combined sluice-pump station project," Transactions of the Chinese Society of Agricultural Engineering, vol. 31, no. 7, pp. 100-108, 2015.

[9] X. He, Y. Zhang, C. Wang et al., "Influence of critical wall roughness on the performance of double-channel sewage pump," Energies, vol. 13, no. 2, p. 464, 2020.

[10] C. Wang, X. Chen, N. Qiu, Y. Zhu, and W. Shi, "Numerical and experimental study on the pressure fluctuation, vibration, and noise of multistage pump with radial diffuser," Journal of the Brazilian Society of Mechanical Sciences and Engineering, vol. 40, no. 10, p. 481, 2018.

[11] H. L. Wang, B. Long, Y. Yang, Y. Xiao, and C. Wang, "Modelling the influence of inlet angle change on the performance of submersible well pumps," International Journal of Simulation Modelling, vol. 19, no. 1, pp. 100-111, 2020.

[12] H. Wang, Z. Qian, D. Zhang, T. Wang, and C. Wang, "Numerical study of the normal impinging water jet at different impinging height, based on wray-agarwal turbulence model," Energies, vol. 13, no. 7, p. 1744, 2020.

[13] L. Zhou, K. Deshpande, X. Zhang, and R. K. Agarwal, "Process simulation of chemical looping combustion using ASPEN plus for a mixture of biomass and coal with various oxygen carriers," Energy, vol. 195, Article ID 116955, 2020.

[14] L. Zhou, W. Wang, J. Hang, W. Shi, H. Yan, and Y. Zhu, "Numerical investigation of a high-speed electrical submersible pump with different end clearances," Water, vol. 12, no. 4, p. 1116, 2020.

[15] L. Zhou, C. Han, L. Bai, W. Shi, and R. Agarwal, "Numerical and experimental study of multiphase transient core-annular flow patterns in a spouted bed," Journal of Energy Resources Technology, vol. 142, no. 9, 2020.

[16] Y. Xu, B. Xi, R. Lu, C. Zhang, Y. Duan, and Y. Zheng, "Influence of hollow rectifying sill on flow pattern in forebay of side-inlet pumping station," International Journal Hydroelectric Energy, vol. 38, no. 7, pp. 161-164, 2020.

[17] L. Shi, J. Zhu, F. Tang, and C. Wang, "Multi-disciplinary optimization design of axial-flow pump impellers based on 
the approximation model," Energies, vol. 13, no. 4, p. 779, 2020.

[18] H. Wang, B. Long, C. Wang, C. Han, and L. Li, "Effects of the impeller blade with a slot structure on the centrifugal pump performance," Energies, vol. 13, no. 7, p. 1628, 2020.

[19] Q. Zhou, G. Fang, X. Wu, Q. Liu, H. Sun, and Y. Wang, "Comprehensive assessment of pump station operation based on genetic projection pursuit model," South-to-North Water Transfers and Water Science \& Technology, vol. 13, no. 5, pp. 985-989, 2015.

[20] Y. Yao and C. Liu, "Pump operation optimization and projection pursuit evaluation strategy based on ant colony optimization," Transactions of the Chinese Society for Agricultural Machinery, vol. 44, no. 3, pp. 38-44, 2013.

[21] J. Zhu, L. Qiang, X. Long, and W. Zhao, "Industrial power load forecasting method based on reinforcement learning and PSO-LSSVM," Engineering Journal of Wuhan University, vol. 11, no. 1, pp. 108-111, 2008.

[22] E. Ahmadebrahimpour, "Optimal operation of reservoir systems using the wolf search algorithm (WSA)," Water Supply, vol. 19, no. 5, pp. 1396-1404, 2019.

[23] X. Feng, Y. Wang, and B. Qiu, "Optimization of pumping station group operation based on optimized parameters using hybrid wolf pack algorithm," Transactions of the Chinese Society of Agricultural Engineering, vol. 36, no. 3, pp. 30-36, 2020.

[24] T. Wang, C. Li, and W. Liu, "Optimal scheduling of surface water intake pump stations based on genetic algorithm," International Journal Hydroelectric Energy, vol. 38, no. 6, pp. 89-91, 2020.

[25] Y. Shang, Q. Fan, L. Shang, Z. Sun, and G. Xiao, "Modified genetic algorithm with simulated annealing applied to optimal load dispatch of the three gorges hydropower plant in China," Hydrological Sciences Journal, vol. 64, no. 9, pp. 1129-1139, 2019.

[26] L. DengWei, "Optimization design based on hierarchic genetic algorithm and particles swarm algorithm," Journal of Algorithms \& Computational Technology, vol. 12, no. 3, pp. 217-222, 2018.

[27] F. Buonamici, R. Furferi, L. Governi, A. Marzola, and Y. Volpe, "Scene acquisition with multiple 2D and 3D optical sensors: a PSO-based visibility optimization," Sensors (Basel), vol. 20, no. 6, 2020.

[28] I. H. Dridi, E. B. Alaïa, P. Borne, and H. Bouchriha, "Optimisation of the multi-depots pick-up and delivery problems with time windows and multi-vehicles using PSO algorithm," International Journal of Production Research, vol. 58, no. 14, pp. 4201-4214, 2020.

[29] Q. Ge, C. Guo, H. Jiang et al., "Industrial power load forecasting method based on reinforcement learning and PSOLSSVM," IEEE Transactions on Cybernetics, vol. 9, 2020.

[30] S. Haidong, D. Ziyang, C. Junsheng, and J. Hongkai, "Intelligent fault diagnosis among different rotating machines using novel stacked transfer auto-encoder optimized by PSO," ISA Transactions, vol. 105, 2020.

[31] Q. Qiao, F. Tao, H. Wu, X. Yu, and M. Zhang, "Optimization of a capacitated vehicle routing problem for sustainable municipal solid waste collection management using the PSOTS algorithm," International Journal of Environmental Research and Public Health, vol. 17, no. 6, 2020.

[32] J. Zhou, Y. Li, G. Xiao, and Y. Zhang, "Multi-objective optimal dispatch of cascade hydropower stations based on shuffled particle swarm operation algorithm," Journal of Hydraulic Engineering, vol. 41, no. 10, pp. 1212-1219, 2010.
[33] L. Fan and N. Xu, "Experimental investigation of fibre particles in a turbulent stirred tank with DPIV," Powder Technology, vol. 316, pp. 329-337, 2017.

[34] I. Lakshmi Mallika, D. V. Ratnam, S. Raman, and G. Sivavaraprasad, "Machine learning algorithm to forecast ionospheric time delays using global navigation satellite system observations," Acta Astronautica, vol. 173, pp. 221231, 2020.

[35] L. Bo, Y. Zhang, W. Zhao, and P. Li, "Novel 3D point set registration method based on regionalized gaussian process map reconstruction," Frontiers of Information Technology \& Electronic Engineering, vol. 21, no. 5, pp. 760-777, 2020.

[36] F. Wang, D. Li, Y. Dong, and W. Wang, "Prediction of building settlements due to earthquake based on gauss process regression model," Science Technology and Engineering, vol. 20, no. 16, pp. 6666-6671, 2020.

[37] S. He, W. Chen, X. Mu, and W. Cui, "Constrained optimization model of the volume of initial rainwater storage tank based on ANN and PSO," Environmental Science and Pollution Research, vol. 27, no. 17, pp. 21057-21070, 2020.

[38] J. Zahiri and H. Nezaratian, "Estimation of transverse mixing coefficient in streams using M5, MARS, GA, and PSO approaches," Environmental Science and Pollution Research, vol. 27, no. 13, pp. 14553-14566, 2020.

[39] S. Tang, S. Yuan, and Y. Zhu, "Deep learning-based intelligent fault diagnosis methods toward rotating machinery," IEEE Access, vol. 8, pp. 9335-9346, 2019.

[40] S. Tang, S. Yuan, and Y. Zhu, "Convolutional neural network in intelligent fault diagnosis toward rotatory machinery," IEEE Access, vol. 8, pp. 86510-86519, 2020.

[41] S. Tang, S. Yuan, and Y. Zhu, "Data preprocessing techniques in convolutional neural network based on fault diagnosis towards rotating machinery," IEEE Access, vol. 8, pp. 149487-149496, 2020.

[42] Y. Zhang, G. Su, and L. Yan, "Cooperative optimization algorithm based on particle swarm optimization and Gaussian process," Systems Engineering and Electronics, vol. 35, no. 6, pp. 1342-1347, 2013.

[43] S. Lijian, Z. Wenpeng, J. Haifeng et al., "Numerical simulation and experimental study on the comparison of the hydraulic characteristics of an axial-flow pump and a full tubular pump," Renewable Energy, vol. 153, 2020.

[44] D. Zhang, W. Jiao, L. Cheng et al., "Experimental study on the evolution process of the roof-attached vortex of the closed sump," Renewable Energy, vol. 164, pp. 1029-1038, 2021.

[45] F. E. Fish, T. Wiliams, and T. Wei, "Tail stands in dolphins: experimental measurement of force generation using bubble DPIV," Integrative and Comparative Biology, vol. 57, p. E261, 2017.

[46] L. F. Han, Y. Cong, X. B. Liu, and C. F. Fu, "Flow velocity field measurement of vertical upward oil-water two-phase immiscible flow using the improved DPIV algorithm based on ICP and MLS," Applied Sciences-Basel, vol. 9, no. 16, 2019.

[47] J. Long and T. H. New, "A DPIV study on the effects of separation distance upon the vortical behaviour of jet-cylinder impingements," Experiments in Fluids, vol. 56, no. 7, 2015.

[48] X. Yang, S. Liu, L. Zhang, J. Su, and T. Ye, "Design and analysis of a renewable energy power system for shale oil exploitation using hierarchical optimization," Energy, vol. 206, Article ID 118078, 2020. 